\title{
Apoptose et sénescence
}

\author{
Marie-Anne Phelouzat, Rafael A. Quadri, Jacques J. Proust
}

Au cours de la sénescence, une diminution du nombre des cellules est observée dans la majorité des organes, résultat du ralentissement de la croissance cellulaire ; de plus, une élimination par apoptose

pourrait être en cause. Celle-ci pourrait être la conséquence d'une carence en facteurs de croissance, d'anomalies de la transmission des signaux interet intracellulaires et de modification du cycle cellulaire. L'apoptose intervient probablement dans l'élimination de cellules sénescentes altérées. Elle pourrait, aussi, être perturbée, entraînant l'élimination de cellules saines et la préservation de cellules anormales. De telles perturbations seraient alors un facteur de susceptibilité aux tumeurs et d'aggravation de la raréfaction des cellules intactes.

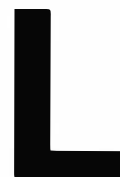

e développement des organismes multicellulaires et leur homéostasie au stade adulte est le résultat d'un contrôle précis et coordonné des processus de prolifération, de différenciation et de mort cellulaire. Au cours du vieillissement, cet équilibre délicat entre prolifération et mort est perturbé. En effet, deux événements sont universellement liés à la sénescence: la diminution du potentiel prolifératif des cellules $\left(m / s n^{\circ} 4\right.$, vol. 1, p. 205) [1] et la disparition cellulaire. Cette raréfaction cellulaire organes. Elle est particulièrement évidente au niveau de la peau, des muscles, des os, des reins, du cerveau [2] et du sang [3] et s'accompagne d'une augmentation de la concentration d'ADN dans le plasma des personnes âgées [4]. Ce phénomène de disparition cellulaire lié à l'âge pourrait être de nature apoptotique [5]. En effet, les progrès récents réalisés dans la compréhension des bases biologiques de la sénescence laissent à penser que les mécanismes d'induction de l'apoptose sont sollicités par certaines modifications physiopathologiques liées au vieillissement. Le lien entre apoptose et vieillissement reste cependant intuitif et encore totalement inexploré [6]. Le but de cet article est d'étayer l'hypothèse de l'origine apoptotique de la délétion cellulaire liée à l'âge en mettant en évidence, dans différents modèles cellulaires, les connexions multiples entre les mécanismes moléculaires de l'apoptose et ceux de la sénescence.

\section{Privation de facteurs de croissance et/ou de survie}

A l'exception des blastomères, les cellules de mammifères ne survivent que grâce aux signaux délivrés par des molécules exogènes produites par d'autres cellules [7]. La privation de facteurs de croissance et/ou de survie représente donc une voie logique d'induction de l'apoptose: une cellule pourrait activer son programme d'autodestruction parce qu'elle n'a pas reçu le signal de survie auquel l'expression de récepteurs appropriés l'avait préparée [8]. Le nombre de cellules d'un type donné est vraisemblablement contrôlé par un phénomène de compétition pour le signal de survie: la diminution de concentration d'un facteur de survie particulier ne pourrait permettre le maintien que d'un nombre limité de cellules. C'est ainsi que l'on explique la régression rapide par apoptose des hyperplasies expérimentales de nombreux organes (cortex surrénalien, foie, rein, pancréas) à la suite de la normalisation des facteurs de croissance correspondants [7].

Au cours du vieillissement, un phénomène analogue pourrait participer au dérèglement des mécanismes homéostatiques chargés du maintien du nombre des cellules de l'organisme. En effet, la synthèse de nombreux facteurs de croissance et/ou de survie décroît avec l'âge et la carence de ces facteurs est susceptible d'activer les mécanismes apoptotiques conduisant à la perte cellulaire. Ce phénomène d'apoptose par privation de facteurs de survie est déjà reconnu, au cours du vieillissement, au niveau de certains organes: l'involution de la glande mammaire et de l'utérus au décours de la ménopause est de nature apoptotique et liée à la privation des stéroïdes sexuels [9]. Les modifications cutanéo-muqueuses sont également liées au déficit de production des œestro- 


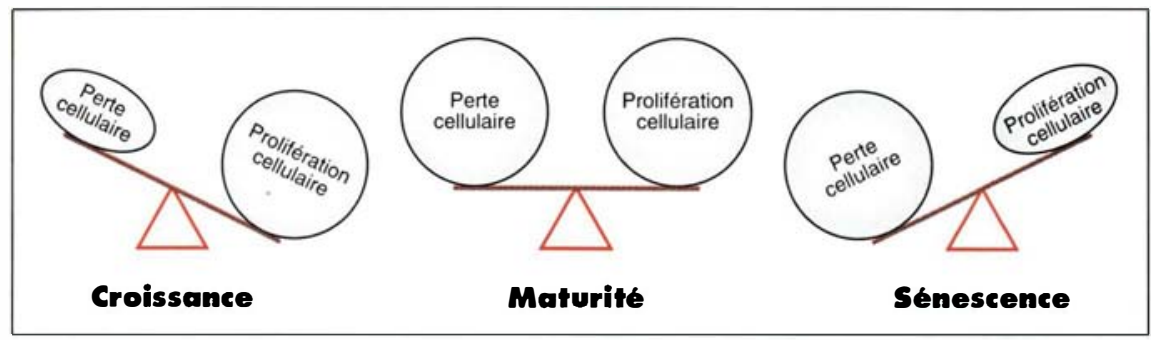

Figure 1. Évolution de l'équilibre entre prolifération et mort cellulaire.

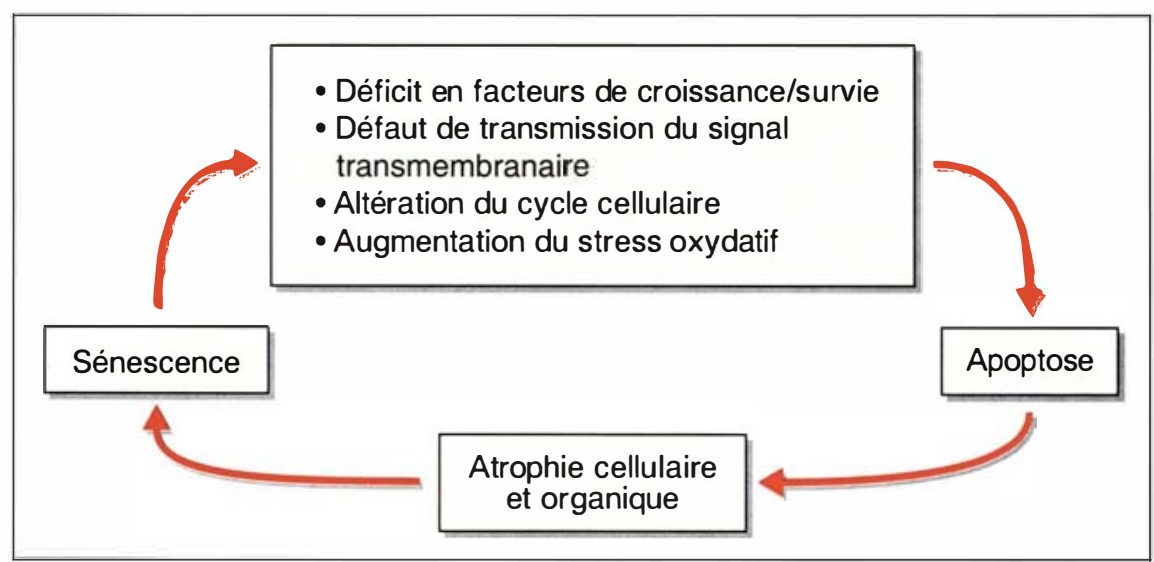

Figure 2. Relation hypothétique entre apoptose et vieillissement.

\begin{tabular}{|c|c|}
\hline \multicolumn{2}{|c|}{$\begin{array}{c}\text { DÉFAUTS DE PRODUCTION DE FACTEURS DE CROISSANCE } \\
\text { ET/OU DE SURVIE ET ATROPHIES ORGANIQUES CLASSIQUEMENT } \\
\text { OBSERVÉS AU COURS DE LA SÉNESCENCE }\end{array}$} \\
\hline $\begin{array}{l}\text { Production hormonale } \\
\text { déficitaire }\end{array}$ & Atrophie organique \\
\hline $\begin{array}{l}\text { Fstrogènes } \\
\text { Androgènes } \\
\text { GH/IGF-1 } \\
\text { Facteurs neurotrophiques } \\
\text { Lymphokines (IL-2) } \\
\text { (?) } \\
\text { DHEA }\end{array}$ & $\begin{array}{l}\text { Peau, muqueuses, glande mammaire, utérus } \\
\text { Peau, muscles, os } \\
\text { Peau, muscles, os, cellules gliales } \\
\text { Neurones, cellules gliales } \\
\text { Compartiment lymphocytaire (?) } \\
\text { Reins } \\
\text { (?) }\end{array}$ \\
\hline
\end{tabular}

$m / s n^{\circ} 6$, vol. 11 , juin 95 gènes [10] et relèvent peut-être du même mécanisme. La diminution de la synthèse des androgènes au cours du vieillissement pourrait être en partie responsable de certaines atrophies organiques et en particulier de la fonte musculaire chez l'homme âgé [11].

La production d'hormone de croissance $(\mathrm{GH})$ et de facteur insulin-like de type I (IGF-I) décline également avec l'âge, parallèlement à la diminution de la masse maigre [12]. L'atrophie musculaire, cutanée et l'ostéopénie observées au cours de la sénescence pourraient être d'origine apoptotique, liées au défaut de production des facteurs de croissance et de survie aux effets pléiotropes que sont la $\mathrm{GH}$ et l'IGF-I. Le fait qu'un traitement hormonal substitutif de six mois par la $\mathrm{GH}$ permette une augmentation significative de la masse maigre, de l'épaisseur cutanée et de la densité osseuse vertébrale chez des personnes âgées de plus de 60 ans présentant un déficit en IGF-I [13], plaide en faveur de l'origine apoptotique de l'atrophie organique de la sénescence.

L'apoptose induite par la privation de facteurs de survie est un phénomène particulièrement bien démontré dans le système nerveux où les neurones meurent en l'absence de facteur de croissance neuronal (NGF) produit par la cellule cible qu'ils innervent [14]. De même, la survie des cellules gliales dépend, selon leur état de maturité et de différenciation, de la présence d'IGF-I, de facteur de croissance dérivé des plaquettes (PDGF), de neurotrophine 3 (NT3) [15] et de facteur neurotrophique ciliaire (CNTF) sécrétés par les neurones qu'ils myélinisent [16]. L'atrophie et la disparition neuronales liées à l'âge pourraient procéder d'une privation de facteurs neurotrophiques [17]. A l'appui de cette hypothèse, il a été démontré que la perfusion intra-thécale de NGF chez le rat Sprague-Dawley âgé entraîne une régression complète de l'atrophie neuronale liée à l'âge, accompagnée d'une amélioration considérable des capacités d'apprentissage $\left(m / s n^{\circ} 3\right.$, vol. $\left.6, p .312\right)[18$, 19]. L'accélération considérable du phénomène de disparition neuro- 
nale au cours de la maladie d'Alzheimer [20] pourrait relever d'un mécanisme similaire, l'accumulation de substance $\beta$-amyloïde empêchant les neurones de recevoir les facteurs neurotrophiques produits par la cellule cible ou par la cellule gliale adjacente [21].

L'apoptose apparaît également comme un des mécanismes régulateurs essentiels de la fonction immunitaire impliqué, aussi bien dans le développement de la réponse immune, que dans le maintien de la mémoire immunologique et l'établissement de la tolérance $\left(\mathrm{m} / \mathrm{s} n^{\circ} 1\right.$, vol. $10, p .125$; $n^{\circ} 1$, vol. $11, p .139$ ) [22]. De ce fait, il est hautement probable que l'apoptose participe aussi pleinement à la dysrégulation du système immunitaire observée au cours du vieillissement. Parmi les différents mécanismes susceptibles d'induire une apoptose non appropriée dans les lymphocytes $\mathrm{T}$ de personnes âgées, la production d'IL2 pourrait représenter un élément critique [23]. En effet, la synthèse du facteur de croissance et de survie qu'est l'IL2 diminue significativement avec l'âge [24]. Par ailleurs, l'incapacité de certains lymphocytes $\mathrm{T}$ activés de produire des quantités adéquates d'IL2 déclenche le processus apoptotique au sein de ces mêmes cellules [25]. Ainsi, au cours du vieillissement, la baisse de production d'IL2 pourrait induire une apoptose lymphocytaire contribuant au déficit immunitaire de la personne âgée.

Le renouvellement et/ou le maintien en vie de très nombreux types cellulaires étant assuré par un réseau paracrine de facteurs de croissance et de survie $\left(m / s n^{\circ} 2\right.$, vol. $11, p$. 301), il est concevable que la mort d'une cellule particulière puisse déclencher un phénomène d'apoptose en chaîne et aboutir à la disparition de toute une unité fonctionnelle cellulaire. Un tel mécanisme pourrait être impliqué dans les pertes cellulaires massives des organismes sénescents.

\section{Défaut de signalisation transmembranaire}

D'une façon générale, la mort par signalisation conduisant à la division cellulaire sont interrompues ou perturbées [26]. Le défaut de transmission des signaux extracellulaires, en particulier des signaux prolifératifs, représente donc un risque mortel pour la cellule.

Un dysfonctionnement lié à l'âge de certains récepteurs membranaires qui, comme le récepteur pour l'antigène des lymphocytes T (TCR), possèdent la propriété de convoyer des signaux de mort et des signaux de survie [22] pourrait, en théorie, transformer un signal prolifératif en un arrêt de mort.

D'autres récepteurs membranaires tels que APO-1/Fas ont la propriété de transmettre un signal apoptotique. Les anticorps dirigés contre les molécules APO-1 ou Fas et leur ligand naturel induisent l'apoptose dans les cellules exprimant ce type de récepteur et pourvues d'un mécanisme de signalisation approprié [22, 27]. Les modalités d'expression de ce récepteur en fonction de l'âge sont encore inconnues. En revanche, dans le système immunitaire, la proportion de lymphocytes CD45 R0 exprimant une forte densité de récepteur Fas, augmente considérablement avec l'âge [28]. L'expression de ce récepteur et l'incapacité des cellules CD45 R0 de produire des quantités adéquates d'IL2 les prédisposent à l'apoptose [25]. Chez les personnes âgées, l'augmentation importante de la proportion des lymphocytes CD45 R0 pourrait jouer un rôle significatif dans le phénomène de l'immunosénescence en raison de la susceptibilité accrue de ces cellules à l'apoptose.

Parmi les autres éléments de la cascade de signalisation, les kinases, que l'on savait impliquées dans la régulation du cycle cellulaire, sont maintenant reconnues comme des éléments importants du mécanisme apoptotique.

Les protéine kinases C (PKC), notamment, semblent jouer un rôle majeur dans l'option prise par la cellule de mourir ou de se diviser. L'activation des PKC par les esters de phorbol permet d'inhiber l'apoptose dans divers systèmes expérimentaux et les inhibiteurs des PKC ont l'effet inverse [29]. Une altération du pro- cessus de signalisation transmembranaire a été mise en évidence, au cours du vieillissement, dans de nombreux modèles cellulaires. Dans les lymphocytes provenant d'organismes âgés, l'altération des mécanismes de transduction des signaux se traduit par un défaut majeur d'activation des PKC [30]. Cette anomalie explique en partie le déficit prolifératif des lymphocytes provenant de donneurs âgés et participe peut-être aussi à leur délétion. Dans ce modèle, un mécanisme par lequel ce défaut d'activation des PKC est susceptible d'induire l'apoptose est encore la privation de facteurs de croissance puisque les PKC activent indirectement certains facteurs de transcription tels que NF$\kappa \mathrm{B}$ et AP-l qui gouvernent l'expression des gènes de l'IL2 et de son récepteur.

Les tyrosine kinases (TK) sont également impliquées dans le processus apoptotique et leur dysfonctionnement peut à la fois provoquer l'arrêt du cycle cellulaire et la mort par apoptose [31]. Au cours du vieillissement, l'activité des TK liées aux récepteurs membranaires diminue notablement [32] et la transmission du signal prolifératif est inadéquate. La cellule pourrait alors opter pour la voie suicidaire plutôt que pour celle de la mitose.

\section{Altération du cycle cellulaire}

Passé le stade de l'ontogenèse, un organisme multicellulaire doit équilibrer prolifération et mort cellulaire, s'il doit maintenir une taille constante. Il n'est donc pas surprenant que les mêmes gènes soient impliqués dans le contrôle du cycle cellulaire et dans celui de l'apoptose [33]. Au cours du vieillissement, il est possible que la modification de l'expression des gènes contrôlant les mécanismes de prolifération [1] déplace l'équilibre prolifération-mort cellulaire en faveur de l'apoptose [34].

Les proto-oncogènes c-fos et c-jun sont exprimés secondairement à l'activation cellulaire et sont également impliqués dans la régulation de l'apoptose [35]. Dans les lymphocytes $T$ activés, dérivés de domneurs 
âgés, l'expression de c-fos est normale alors que l'expression de c-jun est défectueuse [36]. Cette expression différentielle de c-fos et de c-jun avec l'âge retentit sur l'activité du complexe AP-l comportant les produits des gènes jun et fos [37]. L'expression du gène de l'IL2 étant en partie sous le contrôle du complexe de transcription AP-1, le défaut de production d'IL2 constaté au cours du vieillissement [24] est vraisemblablement lié au déséquilibre d'expression de c-fos et de c-jun. Selon le modèle de l'apoptose induite par la privation de facteur de croissance, le défaut d'expression de c-jun lié à l'âge pourrait ainsi aboutir à la mort cellulaire. Dans d'autres modèles cellulaires, l'expression de c-fos et de c-jun est diversement affectée par le vieillissement. Dans les fibroblastes sénescents par exemple, l'expression de c-fos est réprimée juste après le début de la transcription $\left(\mathrm{m} / \mathrm{s} n^{\circ} 3\right.$, vol. 6, p. 313) [38] alors que l'expression de c-jun et de jun $B$ n'est pas modifiée [39]. Selon le type cellulaire, les effets du vieillissement sur le contrôle génétique de l'apoptose peuvent donc être variables.

Le rôle de c-myc est double puisqu'il est à la fois un initiateur de la prolifération et un inducteur de l'apoptose [40]. Son expression, dans des conditions d'arrêt du cycle cellulaire, telles que la privation de facteurs de croissance, entraîne l'apoptose [41]. Les lymphocytes de sujets âgés présentent un défaut d'expression de c-myc qui participe vraisemblablement à la diminution importante du potentiel prolifératif de ces cellules [42, 43]. En toute logique, ce défaut d'expression de c-myc par les cellules provenant d'organismes sénescents devrait rendre ces dernières moins sensibles à l'apoptose. Cependant, la privation simultanée de facteurs de croissance ne permet pas d'augurer du choix final de la cellule entre prolifération et suicide.

Comme c-myc, $p 53$ est un régulateur du cycle cellulaire qui, dans certaines conditions, peut également provoquer l'apoptose. Son expression est induite principalement par les événements susceptibles d'endommager l'ADN [44] mais aussi, dans certains cas, par la privation de facteurs de croissance [45]. Son rôle est alors de bloquer la transition $\mathrm{Gl} / \mathrm{S}$ afin de permettre la réparation des altérations de l'ADN et, le cas échéant, de déclencher l'apoptose. Ce blocage du cycle cellulaire est indirect: en effet, la protéine p53 est un facteur de transcription pour le gène $W A F-1$ dont le produit, la protéine Cipl, est un puissant inhibiteur des kinases dépendantes des cyclines de phase Gl $\left(m / s n^{\circ} 6-7\right.$, vol. 10, p. 744) [46]. Or le gène $W A F-1$, également dénommé sdi 1 (senescent cell-derived inhibitor of DNA synthesis), est surexprimé dans les fibroblastes sénescents ayant perdu leur capacité proliférative et dans les cellules privées de facteurs de croissance [47]. Si elle est confirmée dans d'autres modèles cellulaires, l'hyperexpression de $W A F-1$ pourrait représenter un élément essentiel de la délétion cellulaire et de la perturbation de l'homéostasie proliférative au cours du vieillissement [48].

\section{Bcl-2 et stress oxydatif}

La protéine $\mathrm{Bcl}-2$ est un puissant inhibiteur de l'apoptose dans de nombreux types cellulaires [49]. En particulier, Bcl-2 s'oppose à l'apoptose induite par la privation de facteurs de croissance dans les neurones [50, 51] et dans les lymphocytes [52]. On ignore encore les modalités d'expression de $b c l-2$ en fonction de l'âge. Si l'expression de $b c l-2$, comme celle de nombreux autres gènes [53], décline au cours de la sénescence, la diminution simultanée de la production de facteurs de croissance et de la protéine $\mathrm{Bcl}-2$ pourrait avoir des conséquences catastrophiques sur la survie de certaines populations cellulaires. Une illustration de ce phénomène est fournie par le système immunitaire: la grande susceptibilité des cellules CD45 RO à l'apoptose s'explique en partie par leur faible niveau d'expression de $b c l-2$ [25]. L'augmentation considérable du nombre de ces cellules chez les personnes âgées [28], représente un autre mécanisme susceptible d'impliquer la mort cellulaire dans le dysfonctionnement immunitaire lié à l'âge.
De nombreux agents inducteurs d'apoptose sont, soit des oxydants, soit des activateurs du métabolisme oxydatif cellulaire. A l'inverse, de nombreux inhibiteurs de l'apoptose ont eux-mêmes des propriétés antioxydantes ou augmentent l'efficacité des mécanismes de défense de la cellule contre les radicaux libres oxygénés. En fait, le stress oxydatif apparaît comme l'aboutissement de la plupart des mécanismes inducteurs de l'apoptose [54]. L'influence des radicaux oxygènes dans le processus du vieillissement [55] est maintenant largement reconnue. Les cellules provenant d'organismes vieillissants présentent une extrême vulnérabilité à l'action des radicaux hydroxyles. Les raisons de cette susceptibilité accrue des cellules âgées au stress oxydatif sont encore mal comprises. Quoi qu'il en soit, l'altération des acides nucléiques, des lipides et des protéines par les dérivés oxygénés perturbe le fonctionnement cellulaire et contribue ainsi aux modifications physiopathologiques de la sénescence [56]. Dans ces conditions de débordement des mécanismes anti-oxydants, il serait surprenant que l'activité des radicaux libres produits au cours du vieillissement reste sans effet sur les mécanismes effecteurs de l'apoptose. Là encore, le niveau d'expression de $b c l-2$ est critique puisque le produit de ce protooncogène peut bloquer l'apoptose en s'opposant à l'action des radicaux oxygènes $[57,58]$. Son rôle pourrait être essentiel dans la prévention des pertes neuronales liées à l'âge, puisque le stress oxydatif est à l'origine de plusieurs formes de neurodégénérescence [59] et que $\mathrm{Bcl}-2$ peut empêcher l'apoptose de neurones exposés à l'attaque des radicaux hydroxyles [51]. Cependant, des résultats récents contredisent la notion selon laquelle $\mathrm{Bcl}-2$ pourrait agir en s'opposant aux espèces réactives de l'oxygène. En effet, Bcl-2 a un effet anti-apoptotique en anaérobiose ; il pourrait plutôt agir au niveau de l'action d'enzymes protéolytiques de type ICE $\left(\mathrm{m} / \mathrm{s} n^{\circ} 2\right.$, vol. 10 , p. 232) [60]. 


\section{Conclusion}

La machinerie apoptotique, dont on sait maintenant qu'elle fonctionne bien au-delà de l'organogenèse, pourrait être active dans le processus de la sénescence. Les divers mécanismes susceptibles de conduire à la mort cellulaire par apoptose sont aussi ceux qui sont sollicités et impliqués dans le phénomène du vieillissement. Lors de l'ontogenèse, l'activation du programme de suicide cellulaire permet d'éliminer les cellules devenues inutiles dans l'accomplissement d'une fonction particulière. Au cours du vieillissement, l'apoptose permettrait aussi d'éliminer les cellules au fonctionnement défectueux (apoptose normale). Les mécanismes régulateurs de l'apoptose seraient alors activés par une lésion cellulaire incompatible avec la survie de la cellule. Ainsi la surexpression de $W A F-1$ dans les cellules sénescentes pourrait être induite par des lésions de l'ADN. Dans d'autre cas, l'activation de ces mêmes mécanismes aurait pour conséquence la disparition de cellules normales (apoptose abusive). Par exemple, la diminution progressive de la synthèse de certains facteurs de croissance et/ou de survie avec l'âge ne permettrait le maintien que d'une population cellulaire de plus en plus restreinte. Dans d'autres cas encore, les mécanismes de contrôle de l'apoptose seraient eux-mêmes affectés par le vieillissement et leur activation pourrait résulter en la délétion non appropriée de cellules par ailleurs normales (apoptose aberrante). L'activation inopportune et/ou le dysfonctionnement intrinsèque de la machinerie apoptotique contribuerait ainsi à l'excès de mort cellulaire, phénomène caractéristique de la sénescence

\section{Summary}

Apoptosis and aging

During the adult life, a delicate balance between cell death and cell proliferation ensure that a constant number of cells is maintained throughout the organism. During the course of aging, this homeostatic control is altered and the equilibrium is shifted toward cell death. This results in considerable cell loss in senescent organisms. The true nature of this age-related cell deletion phenomenon could be apoptotic. Indeed, activation of the regulatory mechanisms of apoptosis may occur as a consequence of some pathophysiological alterations

Marie-Anne Phelouzat

Rafael A. Quadri

Jacques J. Proust

Laboratoire d'immunobiologie du vieillissement, institutions universitaires de gériatrie, route de mon idée, 1226 Genève, Thônex, Suisse. known to accompany aging. Namely, diminished synthesis of growth and/or survival factors, transmembrane signaling defects, defaults in the expression of particular genes implicated in cell cycle regulation, and the inability to cope with oxidative stress represent major risk factors liable to induce apoptosis. The untimely activation of biochemical pathways leading to apoptosis would result in excessive cell death, thereby contributing to the characteristic tissular and organismic atrophy observed during the process of senescence

\section{TIRÉS À PART}




\section{RÉFÉRENCES}

1. Finch CE. Gene expression and macromolecular biosynthesis. In : Iongroity, senescence and the genome. London: The University Chicago Press, 1990: 359-463.

2. Bellamy D. Cell death and the loss of structural units of organs. In: Bittles $\mathrm{AH}$, Collins KJ, eds. The biology of human aging. London: Cambridge University Press, 1986 $119-32$.

3. Davy KP, Seals DR. Total blood volume in healthy young and older men. I Appl Physiol $1994 ; 76: 2059-62$

4. Fournié GJ, Martres F, Pourrat JP, Alary C, Rumeau M. Plasma DNA as cell death marker in elderly patients. Gerontology 1993 39: $215-21$.

5. Lockshin RA, Zakeri ZF. Programmed cell death: new thoughts and relevance to aging.J Gerontol 1990 ; 45 : B135-40.

6. Goya RG. Role of programmed cell death in the aging process: an unexplored possibility. Gerontology 1986; 32: 37-42.

7. Raff MC. Social controls on cell survival and cell death. Nature 1992; 356: 397-400.

8. Collins MKL, Perkins GR, Rodriguez-Tarduchy G, Nieto MA, Lopez-Rivas A. Growth factors as survival factors: regulation of apoptosis. BioEssays 1994; 16: 133-8.

9. Bardon S, Vignon F, Montcourrier $P$ Rochefort $H$. Steroid receptor-mediated cytotoxicity of an anticestrogen and an antiprogestin in breast cancer cells. Cancer Res $1987 ; 47: 1441-8$

10. De Ligniere B. Ovarian hormones and cutaneous aging. Rev Fr Gynecol Obstet 1991; $86: 451-4$.

11. Swerdloff RS, W'ang C. Androgens and aging in men. Exp Gerontol 1993; 28: 435-46.

12. Corpas E, Harman SM, Blackman MR. Human growth hormone and human aging. Endocrinol Rev 1993; 14 : 20-39.

13. Rudman D, Feller AG, Nagraj HS, Gergans GA, Lalitha PY, Goldberg AF, Schlenker RA, Cohn L, Rudman IW, Mattson DE. Effect of human growth hormone in men over 60 years old. $N$ Engl J Med 1990; 323: $1-6$.

14. Mesner PW, Winters TR, Green SH Nerve growth factor withdrawal-induced cell death in neuronal PC12 cells resembles that in sympathetic neurons. I Cell Biol 1992 ; $119: 1669-80$.

15. Barres BA, Hart IK, Coles SR, Burne JF, Voyvodic JT, Richardson WD, Raff MC. Cel death and control of cell survival in the oligodendrocytes lineage. Cell 1992; 70: 31-46.

16. Barres BA, Schmid R, Sendnter M Raff MC. Multiple extracellular signals are required for long-term oligodendrocyte survival. Development 1993; 118 : 283-95.

$\mathrm{m} / \mathrm{s} n^{\circ}$ 6, vol. 11, juin 95
17. Finch CE. Neuron atrophy during aging: programmed or sporadic? Trends Neurosci 1993; 16: 104-10.

18. Fisher W, Gage FH, Björklund A. Degenerative changes in forebrain cholinergic nuclei correlate with cognitive impairements in aged rats. Eur I Neurosi 1989; 1 : $34-45$.

19. Fisher $W$, Björklund $A$, Chen $K$, Gage FH. NGF improves spatial memory in aged rodents as a function of age. I Neurosc $1991 ; 11: 1889-906$.

20. Snider WD, Johnson EM. Neurotrophic molecules. Ann Neurol 1989; 26: 489-506.

21. Carson DA, Ribeiro JM. Apoptosis and disease. Lancet $1993 ; 341: 1251-4$.

22. Krammer PH, Behrman I, Daniel P, Dhein J, Debatin KM. Regulation of apoptosis in the immune system. Curr $O p$ Immunol $1994 ; 6: 279-89$.

23. Duke RC, Cohen II. Il-2 addiction: withdrawal of growth factor activates a suicide program in dependent $\mathrm{T}$ cells. Lymphokine Res $1986 ; 5$ : 289-95.

24. Nagel JE, Choppra RK, Chrest FJ, McCoy MT, Schneider EL, Holbrook NJ, Adler WH. Decreased proliferation, interleukin 2 svnthesis, and interleukin 2 receptor expression are accompanied by decreased mRNA expression in phytohemagglutinin-stimulated cells from elderly donors. J Clin Invest 1988; 81 : 1096-102.

25. Salmon M, Pilling D, Borthwick NJ, Viner N, Janossy G, Bacon PA, Akbar AN. The progressive differentiation of primed $T$ cells is associated with an increasing susceptibility to apoptosis. Eur J Immunol 1994; 24 : 892-9.

26. Evans VG. Multiple pathways to apoptosis. Cell Biol Int 1993; 17: 461-76.

27. Golstein P. Deux mécanismes moléculaires pour la cytotoxicité T: perforine/granzymes et Fas. médecine/sciences 1995; 11 : 99 104.

28. Miller RA. Aging and immune function: cellular and biochemical analysis. Exp Gerontol $1994 ; 29: 21-35$

29. Lee S, Christakos S, Small MB. Apoptosis and signal transduction: clues to a molecular mechanism. Curr Op Cell Biol 1993; 5 : 286-91.

30. Proust II, Filburn CR, Harrisson SA Buchholz MA, Nordin AA. Age-related defect in signal transduction during lectin activation of murine $\mathrm{T}$ lymphocytes. $\int \mathrm{Immu}$ nol $1987 ; 139: 1472-8$

31. Chen YY, Rosenberg N. Lvmphoid cells transformed by Abelson virus require the $y$ abl protein-tyrosin kinase only during early Gl. Proc Natl Acad Sci USA 1992; 89: 6683-7.
32. Shi J, Miller RA. Differential tyrosinespecific protein phosphorylation in mouse $T$ lymphocyte subsets : effect of age. J Immunol $1993 ; 151: 730-9$

33. Williams GT, Smith CA. Molecular regulation of apoptosis: genetic controls on cell death. Cell $1993 ; 74$ : $777-9$

34. Kahn A, Briand P. L'apoptose, une mort programmée ou une proliferation avortée? médecine/sciences $1993 ; 9$ : 663-5.

35. Colotta F, Polentarutti N, Sironi M, Mantovani A. Expression and involvement of $c-f o s$ and c-jun protooncogenes in programmed cell death induced by growth factor deprivation in lymphoid cell lines. / Biol Chem 1992; 267 : 18278-83.

36. Song L, Stephens IM, Kittur S, Collins GD, Nagel JE, Pekala PH, Adler WH. Expression of $c$-fos, $c$-jun and jun $B$ in peripheral blood lymphocytes from young and elderly adults. Mech Ageing Dev 1992 ; $65: 149-56$

37. Whisler RL, Beiqing L, Lu LC, Chen M. Reduced activation of transcriptional factor AP-1 among peripheral blood $T$ cells from elderly humans after PHA stimulation: restorative effect of phorbol diesters. Cell Immunol 1993; 152 : 96-109.

38. Seshadri T, Campisi J. Repression of $c$-fos transcription and altered genetic program in senescent human fibroblasts. Science $1990 ; 247: 205-9$

39. Philipps PD, Pignolo RJ. Altered expression of cell cycle dependent genes in senescent WI-38 cells. Exp Gerontol 1992; 27 : 403-7.

40. Piechaczyk M. Myc: le paradoxe d'un oncogène induisant la mort cellulaire programmée. médecine/sciences $1992 ; 8: 586-7$.

41. Evan GI, Wyllie AH, Gilbert CS, Littlewood TD, Land $H$, Brooks $M$, Waters $C M$, Penn LZ, Hancock DC. Induction of apoptosis in fibroblast by $c-M y c$ protein. Cell $1992 ; 69: 119-28$

42. Gamble DA, Schwab R, Weksler ME Szabo P. Decreased steady-state c-myc mRNA in activated $\mathrm{T}$ cell cultures from old humans is caused by a smaller proportion of $\mathrm{T}$ cells that transcribe the c-myc gene. I Immunol $1990 ; 144: 3569-73$

43. Pieri C, Recchioni R, Moroni F, Marcheselli F, Lipponi G. Phytohemagglutinininduced changes of membrane lipid packing, $c-m y c$ and $c-m y b$ encoded protein expression in human lymphocytes during aging. Mech Aging Dev 1992; 64: 177-87.

44. Lu X, Lane DP. Differential induction of transcriptionally active $p 53$ following $\mathrm{UV}$ or ionizing radiation: defects in chromosome instability syndromes? Cell 1993; 75 : 765-78. 


\section{RÉFÉRENCES}

45. Colombel M, Olsson CA, Ng PY, Buttyan R. Hormone-regulated apoptosis results from reentry of differentiated prostate cells onto a defective cell cycle. Cancer Res 1992 ; 52: 4313-9.

46. Marx J. How $p 53$ suppresses cell growth. Science 1993; 262: 1644-5.

47. Noda A., Ning Y, Venable SF, PereiraSmith OM, Smith JR. Cloning of senescent cell-derived inhibitors of DNA synthesis using an expression screen. Exp Cell Res $1994 ; 211: 90-8$.

48. Kihn A. Cycle cellulaire, cancer, sénescence et $p 53$. médecine/sciences 1994; 10 : 206-7.

49. Jacobson MD, Burne JF, King MP, Miyashita T, Reed JC, Raff MC. Bcl-2 blocks apoptosis in cells lacking mitochondrial DNA. Nature 1993; 361 : 365-9.
50. Garcia I, Martinou I, Tsujimoto Y, Martinou JC. Prevention of programmed cell death of sympathetic neurons by the $B C L,-2$ proto-oncogene. Science 1992; 258: 302-4.

51. Zhong LT, Sarafian T, Kane DJ, Charles AC, Mah SP, Edwards RH, Bredesen DE. Bcl-2 inhibits death of central neural cells induced by multiple agents. Proc Natl Acad Sci USA 1993; 90: 4533-7.

52. Deng G, Podack ER Suppression of apoptosis in a cytotoxic T-cell line by interleukin 2- mediated gene transcription and deregulated expression of the proto-oncogène BC.L-2. Proc Nall Acad Sci USA 1993 . $90: 2189-93$

53. Danner DB, Holbrook NJ. Alterations in gene expression with aging. In : Handbook of the biolog' of aging, 3rd ed. London : Academic Press lnc., 1990: 97-115.

54. Buttke TM, Sandstrom PA. Oxydative stress as a mediator of apoptosis. Immunol Tola) 1994; 15:7-10.
55. Harman D. The aging process. Proc Natl Acad Sci USA 1981; 78: 7124-8.

56. Stadtman ER, Oliver CN. Metal-catalyzed oxidation of proteins. Physiological consequences. J Biol Chem 1991 ; 266: 2005-8.

57. Hockenbery DM, Oltvai ZN, Yin XM Milliman CL, Korsmeyer SJ. Bcl-2 functions in an antioxidant pathway to prevent apoptosis. Cell 1993; 75: 241-51.

58. Kahn A. Bcl-2 inhibe-t-il l'apoptose en s'opposant à l'action des radicaux oxygenes? médecine/sciences 1994; 10: 208-9.

59. Coyle JT, Puttfarcken P. Oxydative stress, glutamate, and neurodegenerative disorders. Science 1993; 262: 689-95

60. Martinou J. La mort cellulaire programmée dans le système nerveux. médecine/ sciences $1995 ; 11: 367-73$ 